\title{
High-Precision Tracking of Periodic Signals: a Macro-Micro Approach with Quantized Feedback
}

\author{
Aurélio T. Salton, Jinchuan Zheng, Jeferson V. Flores and Minyue Fu
}

\begin{abstract}
This paper proposes a novel control design method for high-precision positioning systems. The method aims to eliminate the tracking error caused by measurement quantization present in positioning systems with optical encoders. By employing a combined internal model based feedback and quantized feedforward design, we are able to make the output of the positioning system asymptotically track any input signal with one or more sinusoidal components of known frequencies and a possible constant component. When combined with a micro actuator, the resulting dual-stage positioning system is able to track any continuous periodic signal with a known period. Besides theoretical guarantees, the proposed design is validated experimentally and proved able to achieve asymptotic tracking error below $\pm 1 \mu \mathbf{m}$ when subject to a sensor quantization level of $5 \mu \mathrm{m}$.
\end{abstract}

Index Terms-quantization, periodic signals, motion control, macro-micro, dual -stage actuator, tracking.

\section{INTRODUCTION}

D ESIGNERS of positioning control systems always face the tradeoff of positioning accuracy, range of actuation and response time (or bandwidth). For example, voice coil actuators typically have a good range of actuation but their positioning accuracy is often limited by the optical encoder and their response time is limited by the motor dynamics [1]. In contrast, piezo devices can deliver superb dynamic response due to their miniature size and nanometer accuracy due to capacitive sensing but their range of actuation is severely limited [2]-[4].

To overcome this tradeoff, a dual-stage positioning system can be used. Such a system consists of a macro actuator as the primary stage and a piggy-backed micro actuator as the secondary stage. Figure 1 illustrates the schematics of such a design. Due to its relatively larger physical size, the primary stage is able to cover a long range of actuation,

Manuscript received,-- ; revised,-- . This work was partially supported by CNPq (Brazil) under grants 306214/2018-0 and 306223/20180 and Australian Research Council under grant DP200103507. (Corresponding author: Aurélio Salton)

A.T. Salton and J.V. Flores are with the School of Engineering, Universidade Federal do Rio Grande do Sul, Av. Osvaldo Aranha 99, Porto Alegre, RS, Brazil (e-mail: aurelio.salton@ufrgs.br; jeferson.flores@ufrgs.br).

J. Zheng is with the Faculty of Science, Engineering and Technology, Swinburne University of Technology, John St, Hawthorn, VIC 3122, Australia (e-mail: jzheng@swin.edu.au).

$\mathrm{M}$. Fu is with the School of Electrical Engineering and Computer Science, University of Newcastle, Callaghan, NSW 2308, Australia (email: minyue.fu@newcastle.edu.au).

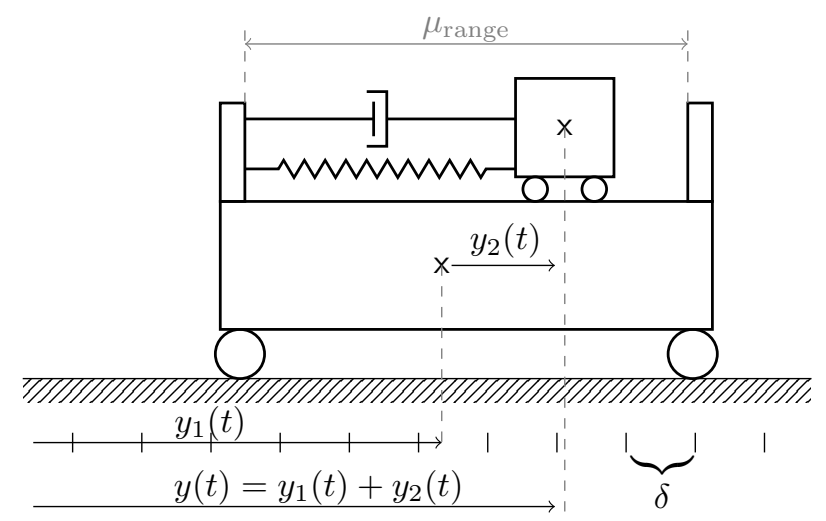

Fig. 1. The macro-micro approach: a micro actuator is connected to the macro (quantized) actuator such that $y(t)=y_{1}(t)+y_{2}(t)$ [14].

but with relatively slower response and coarse resolution. In contrast, the secondary stage is able to respond quickly and accurately, but only over a limited range. A typical combination for translational motion is a linear motor for the primary stage and a piezo device for the secondary stage. Through careful composite control designs, a combined system can take the advantages of both stages, providing fast and high-precision responses over a long working range. Many dual-stage designs are available in the literature; see, e.g., [5][8]. Ample applications of dual-stage positioning systems have been demonstrated; see, e.g., [9]-[13].

Regardless whether the positioning system is single stage or multi-stage, the quantization error of the optical encoder poses a fundamental challenge to the control design. This issue is particularly prominent if the system is designed for long-range high-precision tracking. In this paper, we study a new control design method which focuses on mitigating the quantization error in the feedback measurement, enabling us to significantly improve the tracking accuracy of periodic signals way beyond the accuracy permitted by the optical encoder. When applied in a dual-stage design for tracking periodic signals, the new control design provides the possibility to totally eliminate the tracking error caused by the optical encoder.

Our design method was proposed and tested in our earlier paper [14] for dual-stage positioning systems to track a constant reference input, where we demonstrated how to remove the quantization effect in the primary stage. The work in this paper expands this result to handle the tracking of an arbitrary periodic signal in both single-stage and dual-stage designs. The 
contributions and main results of this paper are summarized below.

- For a single-stage actuator with optical encoder for feedback measurement, we consider the tracking control for any input signal consisting of one or more sinusoidal terms of known frequencies, including a possible constant (DC) term. We propose a novel control design consisting of internal model based feedback together with feedforward quantization and show that the controller can be carefully designed to achieve zero tracking error asymptotically. That is, the quantization effect of the optical encoder in the feedback channel can be completely suppressed.

- For tracking an arbitrary periodic signal with a known period, we deploy a dual-stage system to achieve the task. The primary stage is designed to track an approximate reference signal with a finite number of harmonic terms using the control design above. The secondary stage is designed to track the residual error. Under the assumptions that the secondary stage has negligible measurement error and sufficiently fast dynamic response (which is a reasonable assumption if the secondary stage is a piezo device with capacitive sensing), steady state tracking error can be made arbitrarily small for any continuous periodic input, eliminating the quantization error caused by the optical encoder in the primary stage.

- Arbitrarily small tracking errors are backed up by theoretical proofs, and the superb tracking performance is validated by experimental results. In addition, the theoretical study in the control design offers certain freedoms in the feedback controller which can be used to tune appropriate transient response as well.

The rest of this paper is organized as follows. Section II will provide preliminary material to aid the comprehension of the paper. Section III will provide theoretical results that allow an output quantized actuator to asymptotically track nonstationary trajectories. These results will then be explored in Section IV, by the concept of macro-micro actuators. The proposed strategy will be illustrated by numerical and experimental results in Section V. Conclusions are presented in Section VI.

\section{Problem Statement}

Let us consider a motion system as described by a linear transfer function

$$
G_{1}(s):=\frac{Y_{1}(s)}{U_{1}(s)},
$$

subject to output quantization. Measurements of $y_{1}(t)$ are corrupted by a quantization function defined as

$$
q\left(y_{1}\right):=j \cdot \delta, \quad \forall y_{1} \in \mathcal{Q}_{j},
$$

where $j \in \mathbb{Z}, \delta \in \mathbb{R}^{+}$is called the quantization interval and

$$
\mathcal{Q}_{j}(\delta):=\left\{y_{1} \in \mathbb{R} \mid(j-0.5) \delta \leq y_{1}<(j+0.5) \delta\right\}
$$

is the quantization region.

This paper addresses the problem of tracking a periodic reference $r(t)=r(t+T)$, that is, given measurements $q\left(y_{1}(t)\right)$ achieve $y_{1}(t) \rightarrow r(t)$ as $t \rightarrow \infty$. Two cases are considered: in the first case the reference $r(t)$ is represented by a finite Fourier series with harmonics within the bandwidth of $G_{1}(s)$. Then, given the feedback signal $q\left(y_{1}(t)\right)$, the first problem to be addressed by this paper is that of designing a control law such that the output $y_{1}(t)$ is able to asymptotically track $r(t)$.

In the second case $r(t)$ does not satisfy the assumption above, and a macro-micro approach is proposed. The reference is split into $r(t)=r_{1}(t)+r_{2}(t)$, and the problem becomes that of designing a control law such that the first $m$ harmonics of $r(t)$ are tracked by $y_{1}(t)$, that is, $y_{1}(t) \rightarrow r_{1}(t)$, and such that the higher-order harmonics are tracked by the so-called micro actuator $y_{2}(t) \rightarrow r_{2}(t)$, where:

$$
G_{2}(s):=\frac{Y_{2}(s)}{U_{2}(s)} .
$$

A traditional macro-micro structure is depicted in Fig. 1 and arises from the addition of a micro actuator to the quantized motion system - an additional sensor is also added able to measure the relative displacement $y_{2}(t)$. When compared to the quantized system (henceforth also called the macro actuator), the micro actuator has both a smaller range and a higher bandwidth. Two advantages are obtained by this structure: 1) given its higher bandwidth, the micro actuator is able to track the higher-order harmonics $r_{2}(t)$ in $r(t)$, and 2) given its reduced range ( $\mu_{\text {range }}$ in Fig. 1), measurement errors of $y_{2}(t)$ are negligible when compared to quantization ones $e_{q}(t)=q\left(y_{1}(t)\right)-y_{1}(t)$. As such, even if asymptotic tracking of $r_{2}(t)$ may be impossible for some references, a significantly improved accuracy is achieved by the macro-micro structure provided the higher harmonics in $r_{2}(t)$ are within the micro actuator range.

The overall strategy is depicted in Fig. 2 where, as in Fig. 1, it is clear that the total output is now given by $y(t)=y_{1}(t)+y_{2}(t)$. The periodic reference $r(t)$ is accordingly split as $r(t)=r_{1}(t)+r_{2}(t)$, and two quantization blocks are present: an intrinsic one in the feedback path of $y_{1}(t)$, and the artificial one in the feedforward path of $r_{1}(t)$. We will now proceed by focusing on the tracking control problem for the macro actuator, and later show how to include the micro one in order to achieve an improved performance.

\section{MACRO-StAGE DEsign}

Let the input signal $r_{1}(t)$ be of the following form:

$$
r_{1}(t)=\delta_{0} a_{0}+\sum_{i=1}^{m} a_{i} \sin \left(\omega_{i} t+\theta_{i}\right) .
$$

where $\delta_{0}=0$ or $1, m \geq 1, \omega_{i}>0$ and $a_{i} \neq 0$ for all $i$. Note, in particular, that $r_{1}(t)$ contains at least one sinusoid.

Remark 1: The above structure for $r_{1}(t)$ allows the given reference signal to be one or more sinusoids dislocated by $a_{0}$. This permits good approximation of any periodic reference signal by having a sufficient number of sinusoids. As we will see later in the dual-stage design, for any given periodic reference input signal, (5) can be used as the approximated reference input for the primary stage, leaving the small approximation error to be handled by the secondary stage. We 


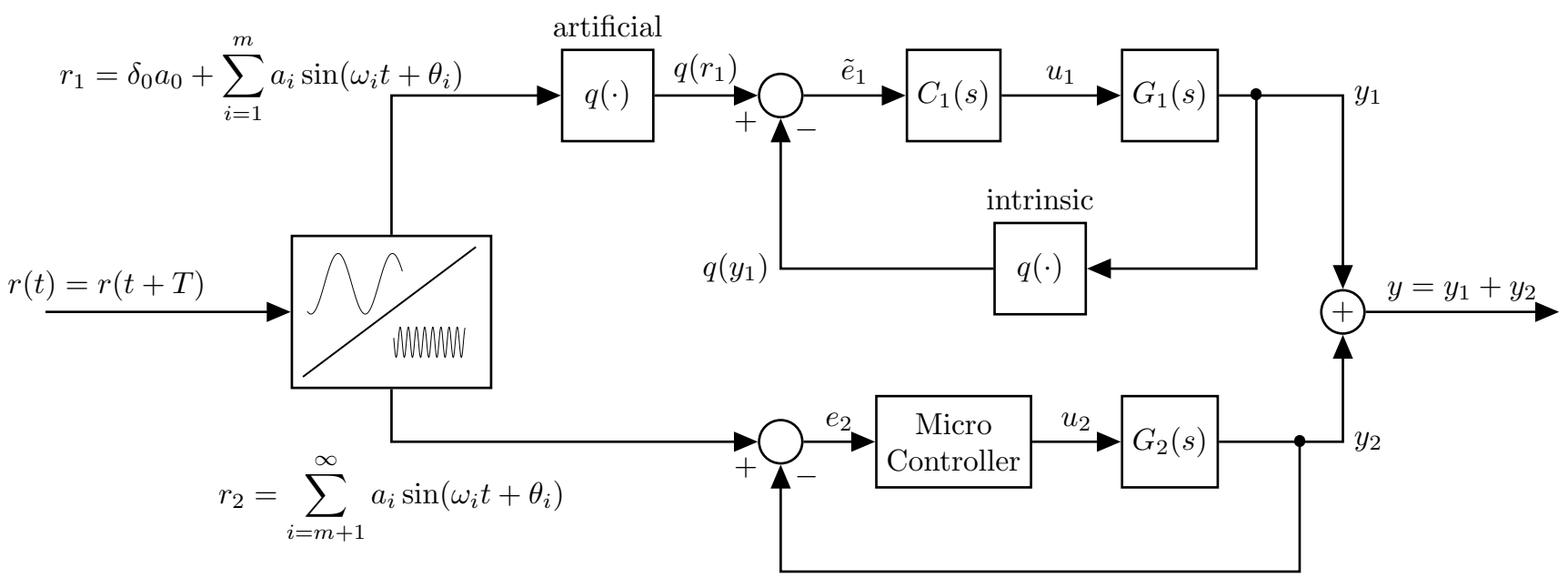

Fig. 2. Schematic representation of the proposed strategy: the reference $r_{1}(t)$ is artificially quantized and asymptotically tracked; the micro actuator is then used to compensate for the residual signal $r_{2}(t)=r(t)-r_{1}(t)$.

also note that (5) also allows quasi-periodic reference signals where $\omega_{i}, i>1$, are not necessarily the harmonics of $\omega_{1}$.

Assumption 1: The magnitude range of $r_{1}(t)$ is sufficiently large with respect to the quantization step size $\Delta$ such that $q\left(r_{1}(t)\right)$ has at least $2 m+\delta_{0}$ different quantization values.

Lemma 1: Under Assumption 1, $r_{1}(t)$ is unique in the sense that for any $\tilde{r}_{1}(t)$ of the form

$$
\tilde{r}_{1}(t)=\delta_{0} \tilde{a}_{0}+\sum_{i=1}^{m} \tilde{a}_{i} \sin \left(\omega_{i} t+\tilde{\theta}_{i}\right)
$$

with $q\left(\tilde{r}_{1}(t)\right)=q\left(r_{1}(t)\right)$ for all $t$, then, $\tilde{r}_{1}(t)=r_{1}(t)$ for all $t$.

Proof: It is easy to see that $r_{1}(t)$ in (5) has only $2 m+\delta_{0}$ free parameters (i.e., all $a_{i}$ and $\theta_{i}$ ). Therefore, it is not possible to have two different functions of the same form with the identical at least $2 m+\delta_{0}$ different values.

Consider the open-loop transfer function $H(s)=C(s) G(s)$ from $\tilde{e}_{1}(t):=q\left(r_{1}(t)\right)-q\left(y_{1}(t)\right)$ to $y_{1}(t)$ as given by

$$
\begin{aligned}
H(s)= & \delta_{0} \frac{k_{0}}{s}+\sum_{i=1}^{m} \frac{k_{i} s}{s^{2}+\omega_{i}^{2}} \\
& +\sum_{i=m+1}^{m+p} \frac{k_{i}}{s+p_{i}}+\sum_{i=m+p+1}^{m+p+q} \frac{k_{i}\left(s+z_{i}\right)}{s^{2}+c_{i} s+d_{i}} .
\end{aligned}
$$

Assumption 2: Suppose the following conditions hold:

- $k_{i}>0$ for all $i=0,1, \ldots, m+p+q$.

- $p \geq 0 ; p_{i}>0$ for all $i=m+1, \ldots, m+p$.

- $q \geq 0 ; c_{i}>0, d_{i}>0$ and $0 \leq z_{i} \leq c_{i}$ for all $i=$ $m+p+1, \ldots, m+p+q$.

The above assumption implies that the first-order terms $(i=$ $m+1, \ldots, m+p)$ and second-order terms $(i=m+p+$ $1, \ldots, m+p+q)$ are strictly Hurwitz. Note that these terms can be void because, as we see below, their presence does not influence the asymptotic behaviour of the closed-loop system. However, the inclusion of these terms can be beneficial for shaping the transient response, offering some design flexibility, and accommodating the plant dynamics.

We have the following main result.
Theorem 1: Suppose $r_{1}(t)$ and $H(s)$ are as in (5) and (6), respectively, and that Assumptions 1-2 hold. Then, the closedloop system is stable and the tracking error $e_{1}(t)=r_{1}(t)-$ $y_{1}(t) \rightarrow 0$ as $t \rightarrow \infty$.

Proof: Define the input components as

$$
\begin{aligned}
& r_{1,0}(t)=a_{0}, \\
& r_{1, i}(t)=a_{i} \sin \left(\omega_{i} t+\theta_{i}\right), i=1, \ldots, m,
\end{aligned}
$$

and the open-loop sub-systems as

$$
\begin{aligned}
H_{1,0}(s) & =\frac{k_{0}}{s}, \\
H_{1, i}(s) & =\frac{k_{i} s}{s^{2}+\omega_{i}^{2}}, i=1, \ldots, m, \\
H_{1, i}(s) & =\frac{k_{i}}{s+p_{i}}, i=m+1, \ldots, m+p, \\
H_{1, i}(s) & =\frac{k_{i}\left(s+z_{i}\right)}{s^{2}+c_{i} s+d_{i}}, i=m+p+1, \ldots, m+p+q .
\end{aligned}
$$

Let the output of each sub-system be $y_{i}(t), i=1, \ldots, m+$ $p+q$. We have

$$
\begin{aligned}
\dot{y}_{1,0}(t) & =k_{0} \tilde{e}_{1}(t), \\
\ddot{y}_{1, i}(t)+\omega_{i}^{2} y_{1, i}(t) & =k_{i} \dot{\tilde{e}}_{1}(t), i=1, \ldots, m, \\
\dot{y}_{1, i}(t)+p_{i} y_{1, i}(t) & =k_{i} \tilde{e}_{1}(t), \\
& i=m+1, \ldots, m+p, \\
& \left.=m+\dot{\tilde{e}}_{1}(t)+z_{i} \tilde{e}_{1}(t)\right),
\end{aligned}
$$

for $i=m+p+1, \ldots, m+p+q$.

It is easy to verify that

$$
\begin{aligned}
\dot{r}_{1,0}(t) & =0 ; \\
\ddot{r}_{1, i}(t)+\omega_{i}^{2} r_{1, i}(t) & =0, i=1, \ldots, m .
\end{aligned}
$$

We also define

$$
\begin{aligned}
& r_{1, i}(t)=0, i=m+1, \ldots, m+p+q, \\
& e_{1, i}(t)=r_{1, i}(t)-y_{1, i}(t), i=0,1, \ldots, m+p+q .
\end{aligned}
$$


It follows that

$$
\begin{aligned}
\dot{e}_{1,0}(t) & =-k_{0} \tilde{e}_{1}(t), \\
\ddot{e}_{1, i}(t)+\omega_{i}^{2} e_{1, i}(t) & =-k_{i} \dot{\tilde{e}}_{1}(t), i=1, \ldots, m, \\
\dot{e}_{1, i}(t)+p_{i} e_{1, i}(t) & =-k_{i} \tilde{e}_{1}(t), \\
i & =m+1, \ldots, m+p, \\
\ddot{e}_{1, i}(t)+c_{i} \dot{e}_{1, i}(t)+d_{i} e_{1, i}(t) & =-k_{i}\left(\dot{\tilde{e}}_{1}(t)+z_{i} \tilde{e}_{1}(t)\right), \\
i & =m+p+1, \ldots, m+p+q .
\end{aligned}
$$

Moreover, it is straightforward to verify that

$$
e_{1}(t)=\delta_{0} e_{1,0}(t)+\sum_{i=1}^{m+p+q} e_{1, i}(t) .
$$

Now construct the Lyapunov-like function

$$
V(t)=\sum_{i=0}^{m+p+q} \frac{1}{k_{i}} V_{i}(t)>0,
$$

where each $V_{i}(t)$ is for each sub-system in (7)-(10), as defined and analysed below.

Case 0: $i=0$. For this case, we take

$$
V_{0}(t)=\frac{\delta_{0}}{2}\left(e_{1,0}(t)\right)^{2} .
$$

Taking derivative, we get

$$
\dot{V}_{0}(t)=\delta_{0} \dot{e}_{1,0}(t) e_{1,0}(t)=-k_{0} \delta_{0} e_{1,0}(t) \tilde{e}_{1}(t) .
$$

Case 1: $i=1, \ldots, m$. For this case, we take

$$
V_{i}(t)=\frac{1}{2}\left(e_{1, i}(t)\right)^{2}+\frac{1}{2 \omega_{i}^{2}}\left(\dot{e}_{1, i}(t)+k_{i} \tilde{e}_{1}(t)\right)^{2} .
$$

Similarly, taking derivative, we get

$$
\begin{aligned}
\dot{V}_{i}(t)= & \dot{e}_{1, i}(t) e_{1, i}(t)+\frac{1}{\omega_{i}^{2}}\left(\ddot{e}_{1, i}(t)+k_{i} \dot{\tilde{e}}_{1}(t)\right) \\
& \times\left(\dot{e}_{1, i}(t)+k_{i} \tilde{e}_{1}(t)\right) \\
& =\dot{e}_{1, i}(t) e_{1, i}(t)-e_{1, i}(t)\left(\dot{e}_{1, i}(t)+k_{i} \tilde{e}_{1}(t)\right) \\
& =-k_{i} e_{1, i}(t) \tilde{e}_{1}(t) .
\end{aligned}
$$

Case 2: $i=m+1, \ldots, m+p$. For this case, we take

$$
V_{i}(t)=\frac{1}{2}\left(e_{1, i}(t)\right)^{2}
$$

Again, taking derivative, we get

$$
\begin{aligned}
\dot{V}_{i}(t) & =\dot{e}_{1, i}(t) e_{1, i}(t) \\
& =\left(-p_{i} e_{1, i}(t)-k_{i} \tilde{e}_{1}(t)\right) e_{1, i}(t) \\
& =-p_{i}\left(e_{1, i}(t)\right)^{2}-\tilde{e}_{1}(t) e_{1, i}(t) \\
& \leq-k_{i} e_{1, i}(t) \tilde{e}_{1}(t) .
\end{aligned}
$$

Case 3: $i=m+p+1, \ldots, m+p+q$. This is the most complicated case. We consider several sub-cases. Denote by $-p_{i 1}$ and $-p_{i 2}$ the two poles of $H_{i}(s)$, i.e., the denominator of $H_{i}(s)$ is expressed as $\left(s+p_{i 1}\right)\left(s+p_{i 2}\right)$. We have $c_{i}=p_{i 1}+p_{i 2}$ and $d_{i}=p_{i 1} p_{i 2}$. By Assumption $2,0 \leq z_{i} \leq p_{i 1}+p_{i 2}$.

Case 3.1: The poles are real and $H_{i}(s)$ has zero-pole cancellation, i.e., $p_{i 1}>0, p_{i 2}>0$ and $z=p_{i 1}$ or $p_{i 2}$. In this sub-case, the simplified $H_{i}(s)=k_{i} /\left(s+p_{i 1}\right)$ or $k_{i} /\left(s+p_{i 2}\right)$.
This is the same as Case 2. So we take $V_{i}(t)$ as in (15) which yields

$$
\dot{V}_{i}(t) \leq-k_{i} e_{1, i}(t) \tilde{e}_{1}(t)
$$

Case 3.2: The poles are real and $0<p_{i 1}<z<p_{i 2}$. Taking partial fraction expansion, $H_{i}(s)$ can be rewritten as

$$
H_{i}(s)=\frac{k_{i 1}}{s+p_{i 1}}+\frac{k_{i 2}}{s+p_{i 2}} .
$$

It is straightforward to check that

$$
k_{i 1}+k_{i 2}=k_{i}, \quad \frac{k_{i 1}}{k_{i}} p_{2}+\frac{k_{i 2}}{k_{i}} p_{1}=z .
$$

Defining $\varepsilon_{i}=k_{i 1} / k_{i}$, we get

$$
\varepsilon_{i}\left(p_{i 2}-p_{i 1}\right)+p_{i 1}=z
$$

Note that the left-hand side above is linear in $\varepsilon$. Hence, $p_{i 1}<$ $z_{i}<p_{i 2}$ implies that $0<\varepsilon_{i}<1$, which in turn implies that both $k_{i 1}>0$ and $k_{i 2}>0$. Therefore, this sub-case goes back to Case 2. We have $e_{1, i}(t)=e_{i 1}(t)+e_{i 2}(t)$, where

$$
\dot{e}_{i j}(t)+p_{i j} e_{i j}(t)=-k_{i j} \tilde{e}(t), \quad j=1,2 .
$$

Take

$$
V_{i}(t)=\frac{k_{i}}{k_{i 1}}\left(e_{i 1}(t)\right)^{2}+\frac{k_{i}}{k_{i 2}}\left(e_{i 2}(t)\right)^{2}
$$

From Case 2, we get

$$
\begin{aligned}
\dot{V}_{i}(t) & \left.\leq-\frac{k_{i}}{k_{i 1}} k_{i 1} e_{i 1}(t) \tilde{e}_{1}\right)-\frac{k_{i}}{k_{i 2}} k_{i 2} e_{i 2}(t) \tilde{e}(t) \\
& =-k_{i} e_{1, i}(t) \tilde{e}_{1}(t) .
\end{aligned}
$$

Case 3.3: The poles are real and either $0 \leq z_{i}<p_{i 1} \leq p_{i 2}$ or $p_{i 2}<z_{i} \leq c_{i}$. Note that this sub-case includes the special case of repeated poles, where $p_{i 1}=p_{i 2}$ and either $0 \leq z_{i}<$ $p_{i 1}$ or $p_{i 1}<z_{i} \leq 2 p_{i 1}$. Define

$$
\begin{aligned}
\rho_{i} & =d_{i}-\left(c_{i}-z_{i}\right) z_{i} \\
& =p_{i 1} p_{i 2}-\left(p_{i 1}+p_{i 2}-z_{i}\right) z_{i} \\
& =\left(z-p_{i 1}\right)\left(z-p_{i 2}\right)
\end{aligned}
$$

Hence, $\rho_{i}>0$ by the conditions of this sub-case. Take

$$
\begin{aligned}
V_{i}(t) & =\frac{1}{2}\left(e_{1, i}(t)\right)^{2} \\
& +\frac{1}{2 \rho_{i}}\left(\dot{e}_{1, i}(t)+\left(c_{i}-z_{i}\right) e_{1, i}(t)+k_{i} \tilde{e}_{1}(t)\right)^{2} .
\end{aligned}
$$


We have

$$
\begin{aligned}
\dot{V}_{i}(t)= & e_{1, i}(t) \dot{e}_{1, i}(t)+\frac{1}{\rho_{i}}\left(\ddot{e}_{1, i}(t)+\left(c_{i}-z_{i}\right) \dot{e}_{1, i}(t)\right. \\
& \left.+k_{i} \dot{\tilde{e}}_{1}(t)\right)\left(\dot{e}_{1, i}(t)+\left(c_{i}-z_{i}\right) e_{1, i}(t)+k_{i} \tilde{e}_{1}(t)\right) \\
= & e_{1, i}(t) \dot{e}_{1, i}(t)+\frac{1}{\rho_{i}}\left(-z_{i} \dot{e}_{1, i}(t)-d_{i} e_{1, i}(t)-k_{i} z_{i} \tilde{e}_{1}(t)\right) \\
\quad & \left(\dot{e}_{1, i}(t)+\left(c_{i}-z_{i}\right) e_{1, i}(t)+k_{i} \tilde{e}_{1}(t)\right) \\
= & e_{1, i}(t) \dot{e}_{1, i}(t) \quad \times\left(\dot{e}_{1, i}(t)+\left(c_{i}-z_{i}\right) e_{1, i}(t)+k_{i} \tilde{e}_{1}(t)\right) \\
& -\frac{1}{\rho_{i}}\left(z_{i} \dot{e}_{1, i}(t)+\left(c_{i}-z_{i}\right) z_{i} e_{1, i}(t)+z_{i} k_{i} \tilde{e}_{1}(t)\right) \\
& \quad-\frac{d_{i}-\left(c_{i}-z_{i}\right) z_{i}}{\rho_{i}} e_{1, i}(t) \\
= & e_{1, i}(t) \dot{e}_{1, i}(t) \\
& -\frac{z_{i}}{\rho_{i}}\left(\dot{e}_{1, i}(t)+\left(c_{1, i}(t)+\left(c_{i}-z_{i}\right) e_{1, i}(t)+k_{i} \tilde{e}_{1}(t)\right)+k_{i} \tilde{e}_{1}(t)\right)^{2} \\
& -e_{1, i}(t)\left(\dot{e}_{1, i}(t)+\left(c_{i}-z_{i}\right) e_{1, i}(t)+k_{i} \tilde{e}_{1}(t)\right) \\
= & -\frac{z_{i}}{\rho_{i}}\left(\dot{e}_{1, i}(t)+\left(c_{i}-z_{i}\right) e_{1, i}(t)+k_{i} \tilde{e}_{1}(t)\right)^{2} \\
& -\left(c_{i}-z_{i}\right)\left(e_{1, i}(t)\right)^{2}-k_{i} e_{1, i}(t) \tilde{e}_{1}(t) \\
\leq & -k_{i} e_{1, i}(t) \tilde{e}_{1}(t) .
\end{aligned}
$$

Case 3.4: Complex poles; $p_{i 1}=\alpha_{i}+j \beta_{i}, p_{i 2}=\alpha_{i}-j \beta_{i}$ with $\alpha_{i}>0$ and $\beta_{i}>0$. We assume $\beta_{i}>0$ because the special case of $\beta_{i}=0$ corresponds to the repeated pole case, which has been considered in Case 3.3. Rewrite (10) as

$$
\ddot{e}_{1, i}(t)+2 \alpha_{i} \dot{e}_{1, i}(t)+\gamma_{i}^{2} e_{1, i}(t)=-k_{i}\left(\dot{\tilde{e}}_{1}(t)+z_{i} \tilde{e}_{1}(t)\right),
$$

where $\gamma_{i}^{2}=\alpha_{i}^{2}+\beta_{i}^{2}$. Note that $0 \leq z_{i} \leq c_{i}=2 \alpha_{i}$ by Assumption 2. Take

$$
\begin{aligned}
V_{i}(t) & =\frac{1}{2}\left(e_{1, i}(t)\right)^{2} \\
& +\frac{1}{2 \eta_{i}^{2}}\left(\dot{e}_{1, i}(t)+\left(2 \alpha_{i}-z_{i}\right) e_{1, i}(t)+k_{i} \tilde{e}_{1}(t)\right)^{2},
\end{aligned}
$$

where, $\eta_{i}^{2}=\left(z_{i}-\alpha_{i}\right)^{2}+\beta_{i}^{2}$. Note that $\eta_{i}^{2}>0$ because $\beta_{i}>0$. Taking the derivative, we get

$$
\begin{aligned}
& \dot{V}_{i}(t) \\
&=\dot{e}_{1, i}(t) e_{1, i}(t)+\frac{1}{\eta_{i}^{2}}\left(\ddot{e}_{1, i}(t)+\left(2 \alpha_{i}-z_{i}\right) \dot{e}_{1, i}(t)+k_{i} \dot{\tilde{e}}_{1}(t)\right) \\
& \times\left(\dot{e}_{1, i}(t)+\left(2 \alpha_{i}-z_{i}\right) e_{1, i}(t)+k_{i} \tilde{e}_{1}(t)\right) \\
&=\dot{e}_{1, i}(t) e_{1, i}(t)+\frac{1}{\eta_{i}^{2}}\left(-z_{i} \dot{e}_{1, i}(t)-\gamma_{i}^{2} e_{1, i}(t)-k_{i} z_{i} \tilde{e}_{1}(t)\right) \\
& \times\left(\dot{e}_{1, i}(t)+\left(2 \alpha_{i}-z_{i}\right) e_{1, i}(t)+k_{i} \tilde{e}_{1}(t)\right) \\
&=\dot{e}_{1, i}(t) e_{1, i}(t) \\
&+\frac{1}{\eta_{i}^{2}}\left(-z_{i} \dot{e}_{1, i}(t)-\left(2 \alpha_{i}-z_{i}\right) z_{i} e_{1, i}(t)-k_{i} z_{i} \tilde{e}(t)\right) \\
& \quad \times\left(\dot{e}_{1, i}(t)+\left(2 \alpha_{i}-z_{i}\right) e_{1, i}(t)+k_{i} \tilde{e}(t)\right) \\
&-\frac{\gamma_{i}^{2}-\left(2 \alpha_{i}-z_{i}\right) z_{i}}{\eta_{i}^{2}} e_{1, i}(t) \xi(t) .
\end{aligned}
$$

with $\xi(t)=\left(\dot{e}_{1, i}(t)+\left(2 \alpha_{i}-z_{i}\right) e_{1, i}(t)+k_{i} \tilde{e}_{1}(t)\right)$. It is easy to verify that

$$
\begin{aligned}
\gamma_{i}^{2}-\left(2 \alpha_{i}-z_{i}\right) z_{i} & =\alpha_{i}^{2}-\left(2 \alpha_{i}-z_{i}\right) z_{i}+\beta_{i}^{2} \\
& =\left(z_{i}-\alpha_{i}\right)^{2}+\beta_{i}^{2} \\
& =\eta_{i}^{2}
\end{aligned}
$$

We have

$$
\begin{aligned}
\dot{V}_{i}(t)= & \dot{e}_{1, i}(t) e_{1, i}(t) \\
& -\frac{z_{i}}{\eta_{i}^{2}}\left(\dot{e}_{1, i}(t)+\left(2 \alpha_{i}-z_{i}\right) e_{1, i}(t)+k_{i} \tilde{e}_{1}(t)\right)^{2} \\
& -e_{1, i}(t)\left(\dot{e}_{1, i}(t)+\left(2 \alpha_{i}-z_{i}\right) e_{1, i}(t)+k_{i} \tilde{e}_{1}(t)\right) \\
= & -\frac{z_{i}}{\eta_{i}^{2}}\left(\dot{e}_{1, i}(t)+\left(2 \alpha_{i}-z_{i}\right) e_{1, i}(t)+k_{i} \tilde{e}_{1}(t)\right)^{2} \\
& -\left(2 \alpha_{i}-z_{i}\right)\left(e_{1, i}(t)\right)^{2}-k_{i} e_{1, i}(t) \tilde{e}_{1}(t) \\
\leq & -k_{i} e_{1, i}(t) \tilde{e}_{1}(t) .
\end{aligned}
$$

Combining all the cases above, we get

$$
\begin{aligned}
\dot{V}(t) & =\sum_{i=0}^{m+p+q} \frac{1}{k_{i}} \dot{V}_{i}(t) \\
& \leq-\left(\delta_{0} e_{i, 0}(t)+\sum_{i=1}^{m+p+q} e_{1, i}(t)\right) \tilde{e}(t) \\
& =-e_{1}(t) \tilde{e}_{1}(t) .
\end{aligned}
$$

Note that $e_{1}(t) \tilde{e}_{1}(t) \geq 0$ because the quantization function is monotonic (i.e., $r_{1}-y_{1} \geq 0 \Rightarrow q\left(r_{1}\right)-q\left(y_{1}\right) \geq 0$ ). This implies that, in steady state, $V(t)$ must be confined in the region where $e_{1}(t) \tilde{e}_{1}(t)=0$, i.e., $e_{1}(t)=0$ or $\tilde{e}_{1}(t)=0$. Also note that $e_{1}(t)=0$ implies $\tilde{e}_{1}(t)=0$. Therefore, this region is equivalent to $\tilde{e}_{1}(t)=q\left(r_{1}(t)\right)-q\left(y_{1}(t)\right)=0$.

Now we focus on the steady state. Since $\tilde{e}_{1}(t)=0$, the steady-state output $y_{1}(t)$ must be of the following form:

$$
y_{1}(t)=\delta_{0} y_{1,0}+\sum_{i=1}^{m} y_{1, i} \sin \left(\omega_{i} t+\psi_{i}\right) .
$$

Note that this is the same form as $r_{1}(t)$. By Lemma 1, $\tilde{e}_{1}(t)=$ $q\left(r_{1}(t)\right)-q\left(y_{1}(t)\right)=0$ for all $t$ in steady state implies that $y_{1}(t)=r_{1}(t)$ in steady state. That is, the tracking error $e(t) \rightarrow$ 0 as $t \rightarrow \infty$.

\section{Dual-stage Design}

The dual-stage design is illustrated in Fig. 2 where the macro and micro actuators are respectively depicted as $G_{1}(s)$ and $G_{2}(s)$.

Instead of tracking the desired periodic reference $r(t)$, the macro actuator will be set in a persistent motion around the reference in a trajectory $r_{1}(t)$ in the form of (5). That is, $r_{1}(t)$ is a periodic signal that consists of at least one sinusoidal term - and a possible DC term - that approximates $r(t)$. The approximation is done such that the residual signal $r(t)-r_{1}(t)$ can be tracked by the secondary stage. In the case that the reference signal $r(t)$ is a constant (i.e., DC only), we refer back to the idea in our earlier paper [14] which makes $r_{1}(t)=$ $r(t)+a_{1} \sin \left(\omega_{1} t\right)$ with a relatively small $a_{1}$ so that $r_{1}(t)$ has 


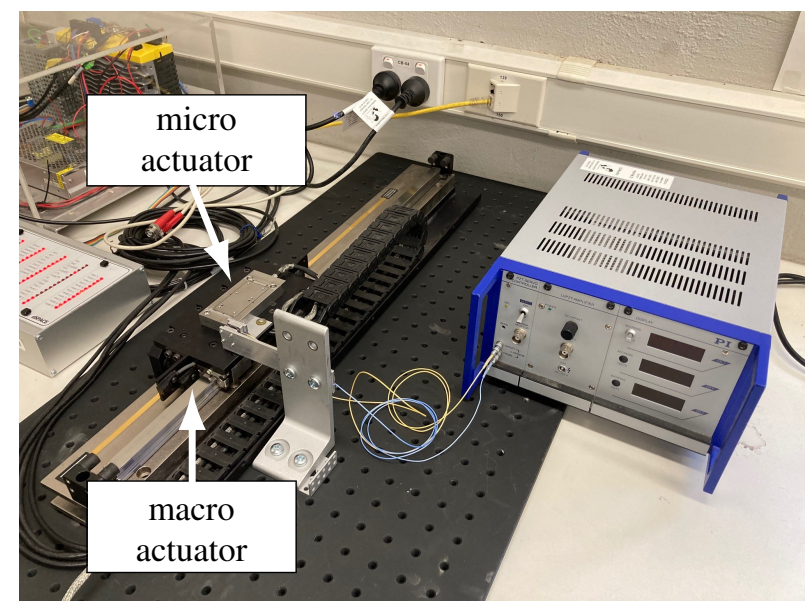

Fig. 3. The experimental macro-micro actuator comprised of a linear motor and a piezoelectric device [8].

a sinusoidal term and the residual signal $r(t)-r_{1}(t)$ can be tracked by the secondary stage as well.

With the above configuration, our proposed control design for the primary stage in the previous section can be applied to ensure asymptotic tracking of $r_{1}(t)$ despite sensor quantization. For a given plant $G_{1}(s)$, it suffices to design the controller $C_{1}(s)$ such that $H_{1}(s)=C_{1}(s) G_{1}(s)$ is in the form of (6). If designing such $C_{1}(s)$ is not possible (for example, if $G_{1}(s)$ has marginal or unstable poles, or if a noncausal controller would be required to match the relative degree of $H_{1}(s)$ ), then design $C_{1}(s)$ such that it is a stabilising controller and $H_{1}(j \omega)$ approximates that in (6) as much as possible for the frequency range of interest. In this case, there may be some small steady-state error.

Since the overall output of the system is now given by $y(t)=y_{1}(t)+y_{2}(t)$, the task of the micro actuator is to compensate for the residual signal by means of

$$
r_{2}(t)=r(t)-r_{1}(t)
$$

from which it follows that $y(t)$ will asymptotically track $r(t)$ if each actuator asymptotically tracks its own reference. The secondary actuator tracking of $r_{2}(t)$ is a standard control problem that may be addressed by traditional methods based on the internal model principal such as, e.g., resonant or repetetive controllers [15], iterative learning [16].

As mentioned earlier, the strictly stable terms $H_{1, i}(s), i=$ $m+1, \ldots, m+p+q$ do not affect the steady state performance. But they can be added to improve transient response and disturbance attenuation. For example, if attenuation is needed around some frequency $\omega_{0}$, we can add a term

$$
H_{1, i}(s)=\frac{k_{i}\left(s+\eta_{i} \alpha_{i}\right)}{\left(s+\alpha_{i}\right)^{2}+\beta_{i}^{2}}
$$

with $\alpha_{i}^{2}+\beta_{i}^{2}=\omega_{0}^{2}$ and a small $\alpha_{i}>0$. Small $\alpha_{i}$ implies that good attenuation is achieved around $\omega_{0}$. These strictly stable terms are very general, except for two restrictions: $k_{i}>0$ for all $i$ and $0 \leq z_{i} \leq c_{i}$ in the second order terms.

\section{Application Examples}

The experimental setup used for the validation of the proposed results, depicted in Fig. 3, is controlled by a DSP system
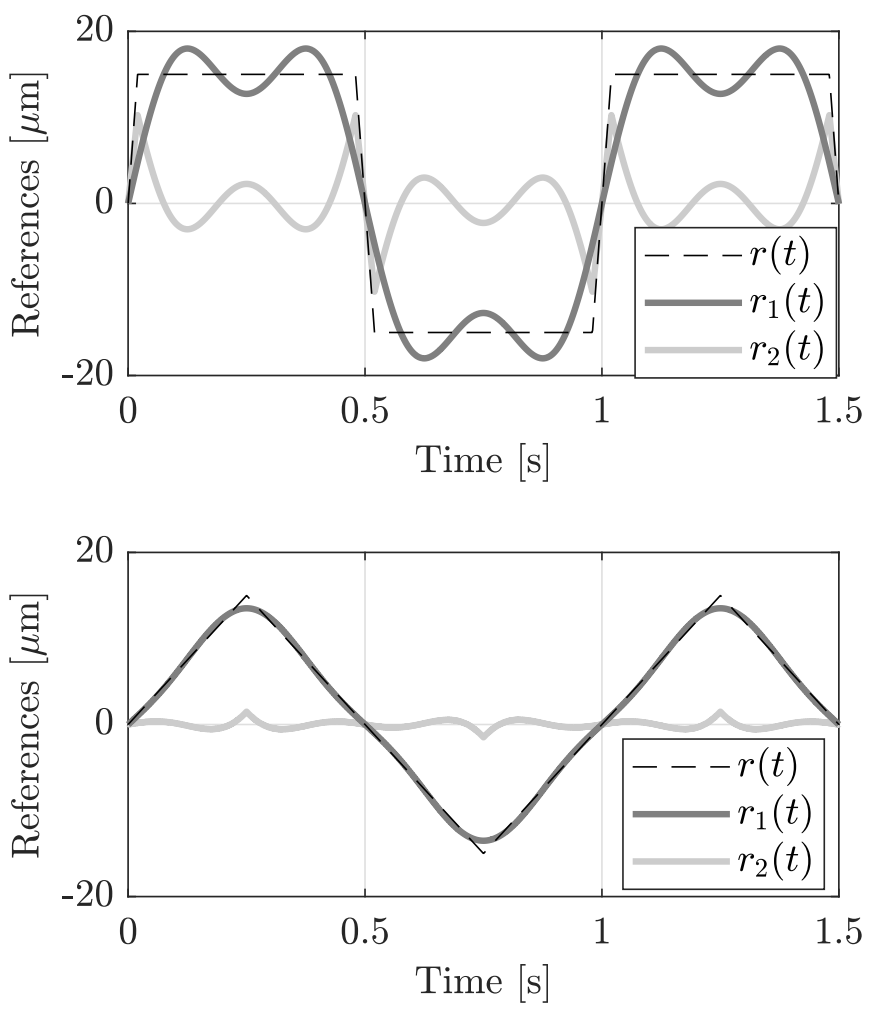

Fig. 4. References considered for tracking: $r_{1}(t)$ is defined as the first two harmonics of $r(t)$, the remaining harmonics are left for $r_{2}(t)=$ $r(t)-r_{1}(t)$.

(dSPACE-DS1103) working at $5 \mathrm{kHz}$. Both outputs $y_{1}(t)$ and $y_{2}(t)$ are measured by capacitive sensors with nanometer resolution from but, in order to illustrate the contribution, an artificial quantization level of $\delta=5 \mu \mathrm{m}$ was applied to $y_{1}(t)$ when used for feedback. This setup was identified in [8] with the macro actuator model described by a mass subject to damping,

$$
G_{1}(s)=\frac{k}{s(s+a)}
$$

with $k=1.7 \times 10^{7}$ and $a=9.42$, and the micro actuator model given by,

$$
G_{2}(s)=\frac{3.032 \times 10^{6}}{s^{2}+1810 s+1.011 \times 10^{6}} .
$$

Triangular and trapezoidal waves with fundamental frequency $\omega_{r}=2 \pi \mathrm{rad} / \mathrm{s}$ described in Fig. 4 will be assigned to $r(t)$, both of which have half wave symmetry and are solely composed of odd harmonics. We will design $C_{1}(s)$ according to (6), so that it tracks the fundamental $\omega_{r}$ and the first odd harmonic $3 \omega_{r}$ of the reference $r(t)$. Therefore, the desired $H_{1}(s)=C_{1}(s) G_{1}(s)$ must encompass the poles of $G_{1}(s)$ and two extra pairs of complex poles:

$$
\begin{aligned}
H_{1}(s) & =\frac{k_{0}}{s}+\frac{k_{1} s}{s^{2}+\omega_{r}^{2}}+\frac{k_{2} s}{s^{2}+\left(3 \omega_{r}\right)^{2}}+\frac{k_{3}}{s+a}, \\
& =\frac{N_{H}(s)}{s\left(s^{2}+\omega_{r}^{2}\right)\left(s^{2}+\left(3 \omega_{r}\right)^{2}\right)(s+a)},
\end{aligned}
$$




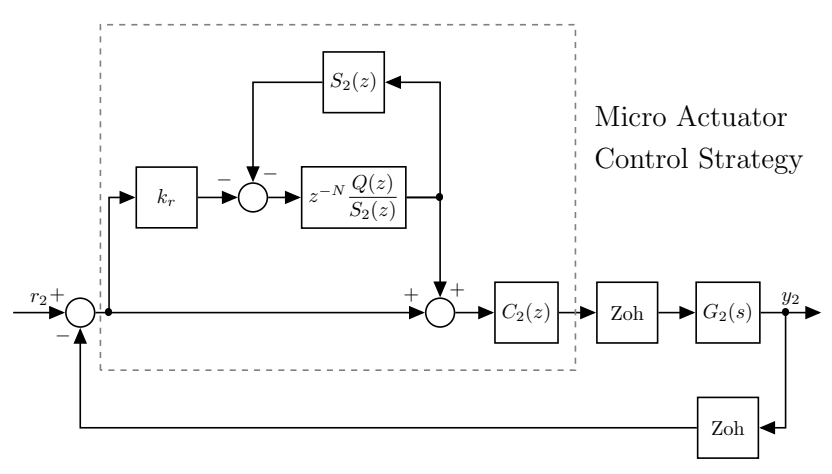

Fig. 5. The plug-in repetitive control strategy applied to the micro actuator micro.

where $N_{H}(s)$ is a fifth degree polynomial

$$
N_{H}(s)=A_{5} s^{5}+A_{4} s^{4}+A_{3} s^{3}+A_{2} s^{2}+A_{1} s+A_{0},
$$

with coefficients,

$$
\begin{aligned}
& A_{5}=k_{0}+k_{1}+k_{2}+k_{3}, \\
& A_{4}=a\left(k_{0}+k_{1}+k_{2}\right), \\
& A_{3}=w_{r}^{2}\left(k_{0} 10+9 k_{1}+k_{2}+10 k_{3}\right), \\
& A_{2}=a w_{r}^{2}\left(10 k_{0}+9 k_{1}+k_{2}\right), \\
& A_{1}=9 w_{r}^{4}\left(k_{0}+k_{3}\right), \\
& A_{0}=k_{0} 9 w_{r}^{4} a .
\end{aligned}
$$

In order to satisfy Assumption 2, it follows that $k_{i}>0$, $i=0, \ldots, 3$ and consequently $A_{j}>0, j=0, \ldots, 5$. Therefore, (22) has relative degree one and, since $G_{1}(s)$ has relative degree two, a noncausal controller would be required to satisfy $C_{1}(s) G_{1}(s)=H_{1}(s)$. To avoid this problem, an extra pole $p_{\bar{\omega}}$ is added to $C_{1}(s)$ which, together with $k_{0}, \ldots, k_{3}$, is chosen so that the closed loop is stable and (22) is satisfied for a large frequency range:

$$
C_{1}(s)=\frac{k_{b w}}{k} \frac{p_{\bar{\omega}}}{s+p_{\bar{\omega}}} \frac{N_{H}(s)}{\left(s^{2}+\omega_{r}^{2}\right)\left(s^{2}+\left(3 \omega_{r}\right)^{2}\right)} .
$$

In the above, $k_{b w}$ and $p_{\bar{\omega}}>\left(3 \omega_{r}\right)^{2}$ are free parameters that respectively set the bandwidth of $C_{1}(s) G_{1}(s)$ and determine the frequency up to which (22) will be valid. These parameters are listed in Table I. Since the control strategy is implemented in discrete time with $T=0.2 \mathrm{~ms}$, the following discrete control (obtained by the matched discretization method) was implemented:

$$
C_{1}(z)=10^{-3} \times \frac{z^{5}-4.99 z^{4}+9.99 z^{3}-9.99 z^{2}+4.99 z-0.998}{z^{5}-4.81 z^{4}+9.27 z^{3}-8.91 z^{2}+4.27 z-0.818}
$$

The micro actuator is left with the task of tracking the remaining harmonics present in $r(t)$. Since triangular and trapezoidal signals have infinite harmonics, so does $r_{2}(t)=$

TABLE I

CONTROL PARAMETERS FOR $C_{1}(s)$.

\begin{tabular}{c|c|c|c|c|c}
$k_{0}$ & $k_{1}$ & $k_{2}$ & $k_{3}$ & $k_{b w}$ & $p_{\bar{\omega}}$ \\
\hline 1 & 10 & 10 & 0.1 & 10 & $10^{3}$
\end{tabular}

$r(t)-r_{1}(t)$, making the repetitive controller strategy a suitable candidate for the micro loop. We have opted for a zero-phase discrete-time odd-harmonic repetitive controller, as depicted in Fig. 5, and followed the design steps in [17]. A discrete-time control law was designed based on traditional loop shaping techniques,

$$
C_{2}(z)=0.25 \frac{z^{2}-1.653 z+0.6896}{(z-1)(z-0.8187)}
$$

and applied together with a zero phase filter $Q(z)=0.44 z^{2}+$ $0.44 z+0.11$. The remaining parameters in Fig. 5 are $S_{2}(z)=$ $C_{2}(z) G_{2}(z) /\left(1+C_{2}(z) G_{2}(z)\right), k_{r}=1.2$ and $N=2500$.

\section{A. Tracking a Trapezoidal Wave}

Here we show the tracking of a symmetric Trapezoidal wave with amplitude $A_{t r}=15 \mu \mathrm{m}$ and slope $a_{s}=0.875 \mu \mathrm{m} / \mathrm{ms}$. The macro actuator is set to track the first two harmonics,

$$
r_{1}(t)=\frac{4 A_{t r}}{\pi}\left(\sin \left(\omega_{r} t\right)+\frac{1}{3} \sin \left(3 \omega_{r} t\right)\right),
$$

and the micro actuator tracks the difference $r_{2}(t)=r(t)-$ $r_{1}(t)$. The respective reference signals are shown in the top plot of Fig. 4, simulation results are depicted in Fig. 6, and experimental results are depicted in Fig. 7. The middle plot of Fig. 6 shows the tracking error, which does not converge to zero due to the approximations in controller $\left.C_{1}(s): 1\right)$ it required the additional pole at $p_{\bar{\omega}}$ in order to achieve causality; 2 ) it was implemented in discrete time with a large sampling time of $T=0.2 \mathrm{~ms}$. Given the above, we were only able to achieve $C_{1}(s) H_{1}(s) \approx H_{1}(s)$. As expected, the resulting error is small, but asymptotic convergence to zero is not achieved.

The experimental results are depicted in Fig. 7 and show a very similar behavior. The error is below the $\pm 1 \mu \mathrm{m}$ mark, representing a small increase from what achieved in simulations. This fact is explained by sensor noise and nonlinear phenomena not represented in the ideal model, such as, backslash and static friction.

\section{B. Tracking a Triangular Wave}

The next signal to be tracked is a Triangular wave with amplitude $A_{t r}=15 \mu \mathrm{m}$ and, as before, $\omega_{r}=2 \pi \mathrm{rad} / \mathrm{s}$. The first two harmonics, set as the macro actuator reference, are now given by

$$
r_{1}(t)=\frac{8 A_{t r}}{\pi^{2}}\left(\sin \left(\omega_{r} t\right)-\frac{1}{9} \sin \left(3 \omega_{r} t\right)\right) .
$$

Reference signals are shown in the bottom plot of Fig. 4, and simulation plots are depicted in Fig. 8. Very similar results to those obtained by the trapezoidal signal are shown. There is a residual error due to approximations in $C_{1}(s)$ both in the simulation and experimental results. Nevertheless, the overall experimental error is well below the $\pm 1 \mu \mathrm{m}$ mark, despite sensor quantization of $\delta=5 \mu \mathrm{m}$. 

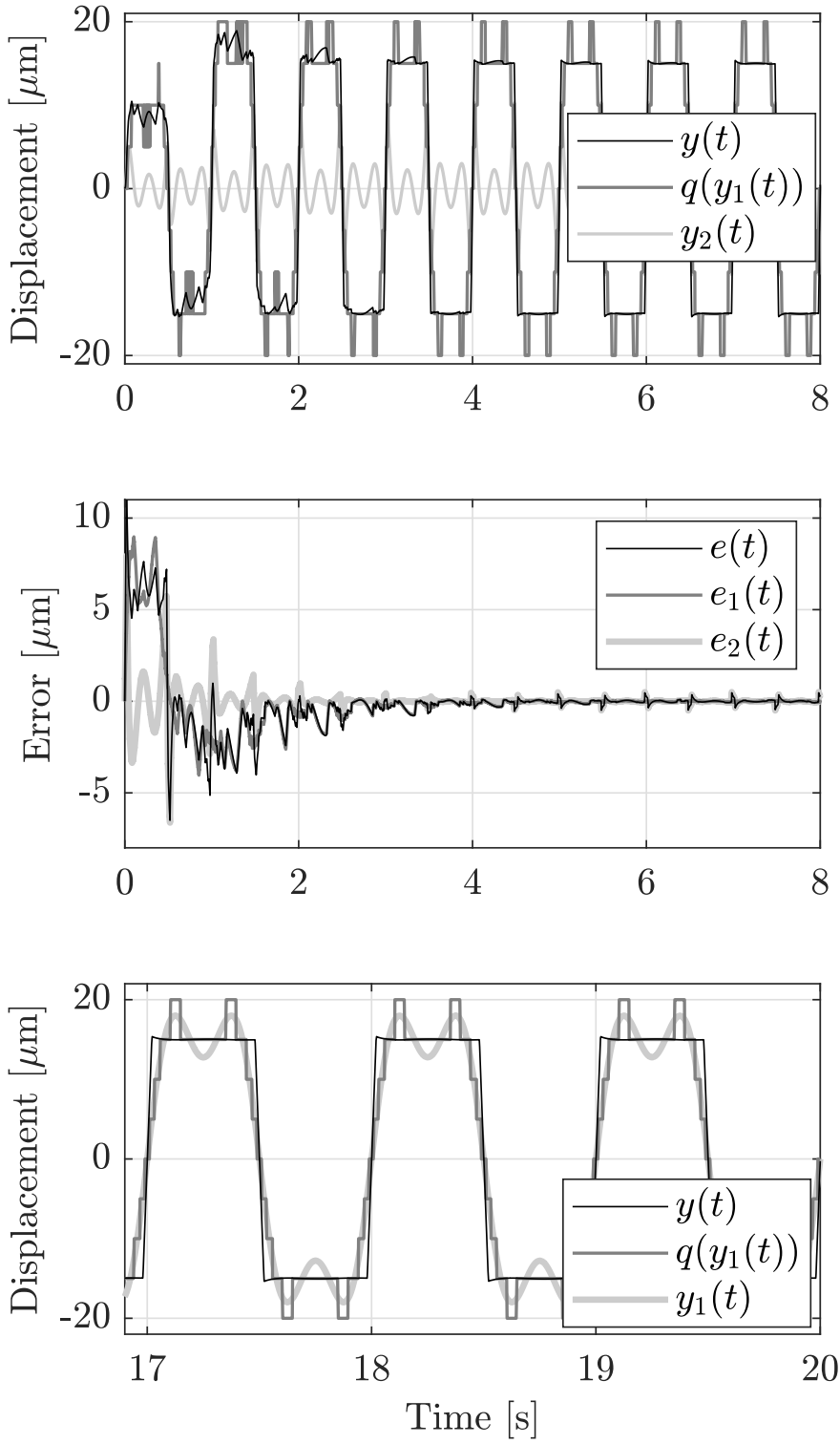

Fig. 6. Simulation results: tracking of a trapezoidal wave. Top plot (outputs): total $y(t)=y_{1}(t)+y_{2}(t)$, quantized $q\left(y_{1}(t)\right.$ and micro actuator $y_{2}(t)$. Middle plot (errors): total $e(t)$, macro $e_{1}(t)=r_{1}(t)-y_{1}(t)$ and micro $e_{2}(t)=r_{2}(t)-y_{2}(t)$. Bottom plot: steady state of the total output $y(t)$, the quantized output $q\left(y_{1}(t)\right.$ used for feedback, and the actual position of $y_{1}(t)$. The overall tracking error converges to the $\pm 0.5 \mu \mathrm{m}$ range, despite the sensor quantization of $\delta=5 \mu \mathrm{m}$.

\section{CONCLUSION}

We have presented a novel control design method for high-precision positioning systems to counter the quantization errors in the feedback channel. In theory, the method is able to eliminate the tracking error for any periodic reference input with a known period. It is also validated experimentally that the tracking error can be suppressed down to the level substantially lower than the quantization error. The proposed method is effective for both single-stage actuators and dual-stage actuators. Future work can aim at generalizing the method to handle a broader class of systems, a wider range of tracking
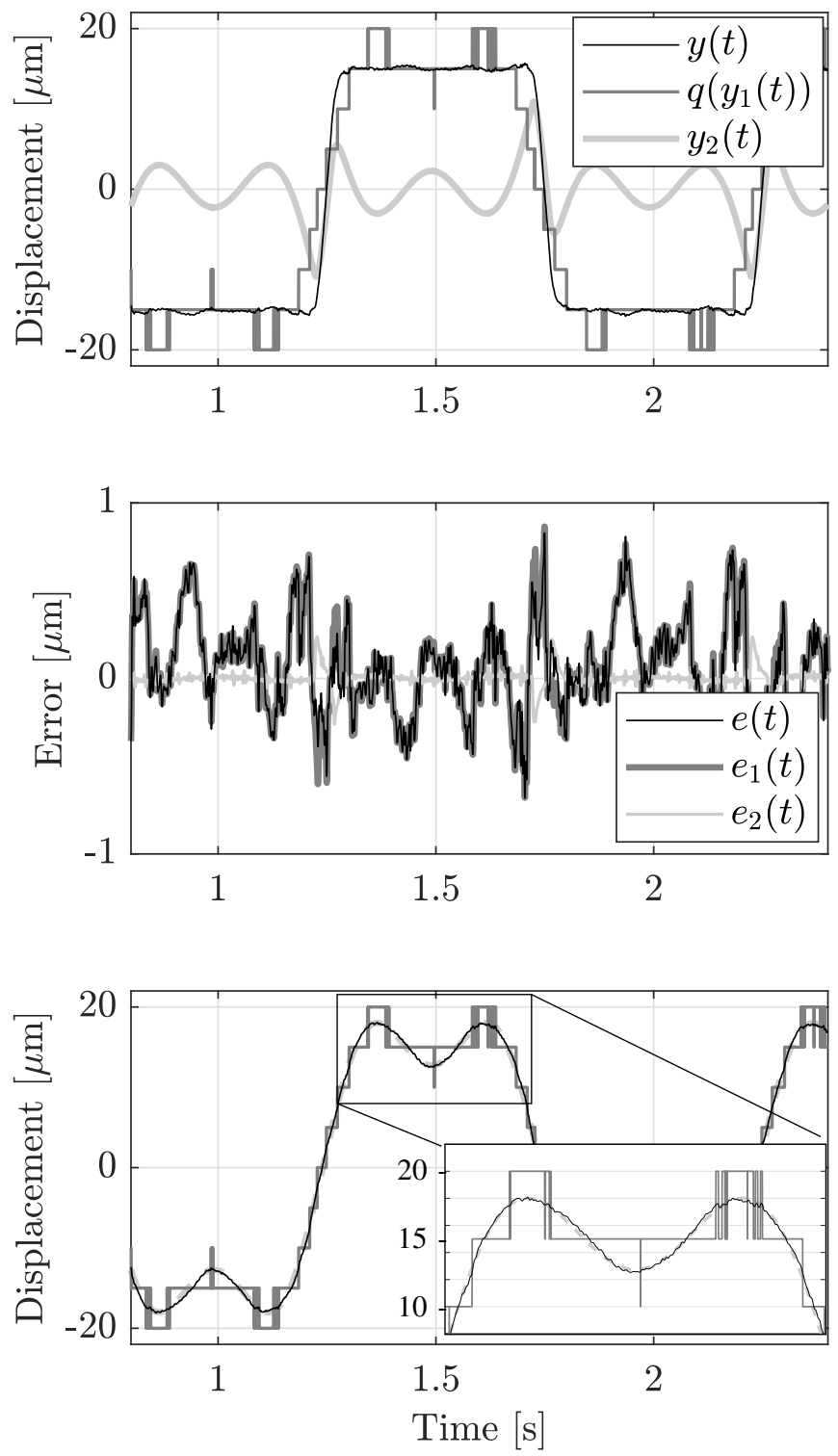

Fig. 7. Experimental results: tracking of a trapezoidal wave. Top and middle plot are as before. Bottom plot: reference $r_{1}(t)$, quantized output $q\left(y_{1}(t)\right.$ used for feedback, and the actual position of $y_{1}(t)$. The overall tracking error is well within the $\pm 1 \mu \mathrm{m}$ range, despite the sensor quantization of $\delta=5 \mu \mathrm{m}$.

problems and some types of measurement uncertainties, such as $\mathrm{AD}$ conversion.

\section{REFERENCES}

[1] S. Devasia, E. Eleftheriou, and S. O. R. Moheimani, "A survey of control issues in nanopositioning," IEEE Transactions on Control Systems Technology, vol. 15, no. 5, pp. 802-823, 2007.

[2] D. Zhang, S. Zhao, Q. Zheng, and L. Lin, "Absolute capacitive grating displacement measuring system with both high-precision and longrange," Sensors and Actuators A: Physical, vol. 295, pp. 11 - 22, 2019.

[3] K. Peng, X. Liu, Z. Chen, Z. Yu, and H. Pu, "Sensing mechanism and error analysis of a capacitive long-range displacement nanometer sensor based on time grating," IEEE Sensors Journal, vol. 17, no. 6, pp. 15961607, March 2017. 

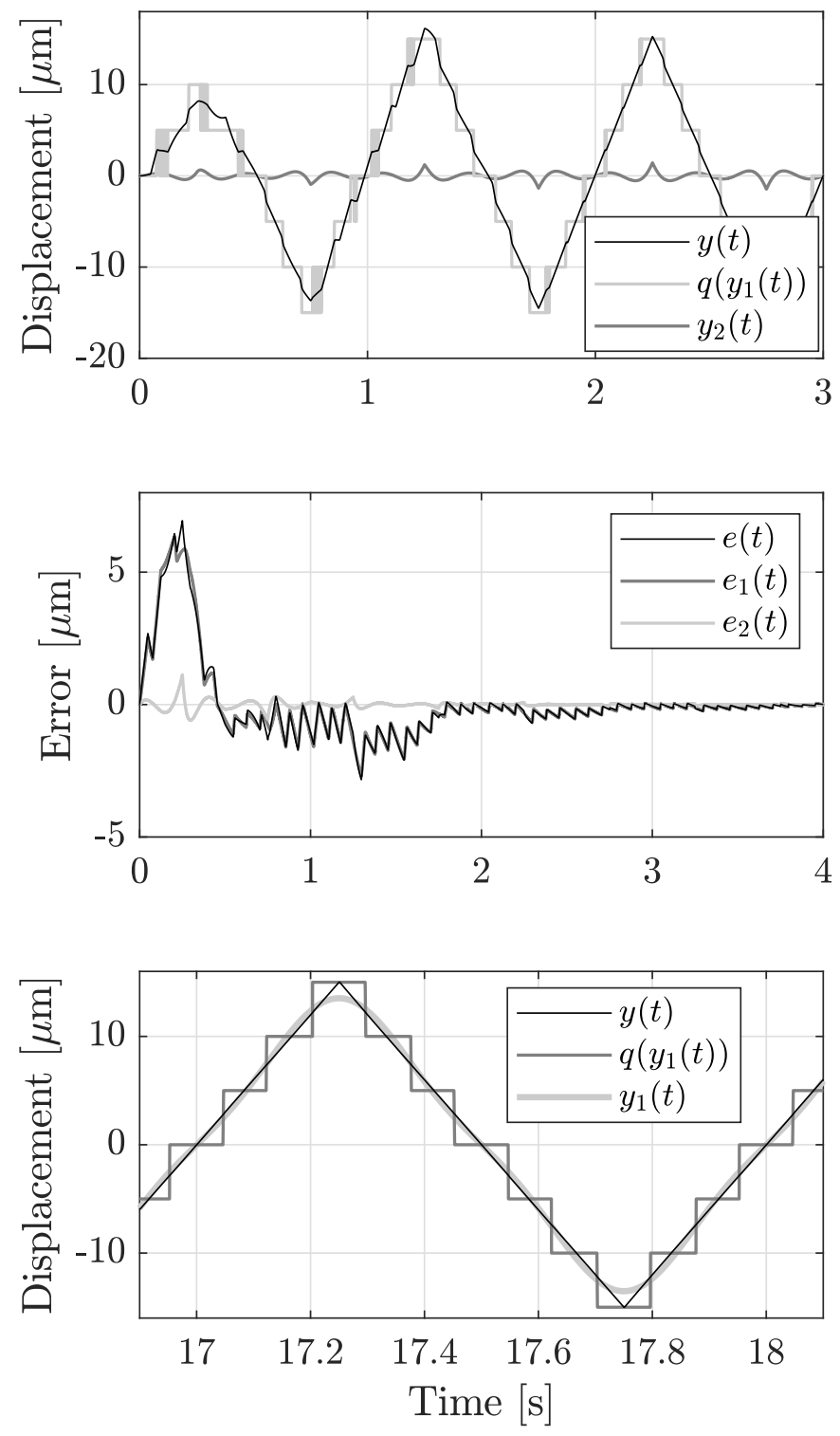

Fig. 8. Simulation results: tracking of a triangular wave. Top plot (outputs): total $y(t)=y_{1}(t)+y_{2}(t)$, quantized $q\left(y_{1}(t)\right.$ and micro actuator $y_{2}(t)$. Middle plot (errors): total $e(t)$, macro $e_{1}(t)=r_{1}(t)-y_{1}(t)$ and micro $e_{2}(t)=r_{2}(t)-y_{2}(t)$. Bottom plot: steady state of the total output $y(t)$, the quantized output $q\left(y_{1}(t)\right.$ used for feedback, and the actual position of $y_{1}(t)$. The overall tracking error converges to the $\pm 0.5 \mu \mathrm{m}$ range, despite the sensor quantization of $\delta=5 \mu \mathrm{m}$.

[4] J. B. Liseli, J. Agnus, P. Lutz, and M. Rakotondrabe, "An overview of piezoelectric self-sensing actuation for nanopositioning applications: Electrical circuits, displacement, and force estimation," IEEE Transactions on Instrumentation and Measurement, vol. 69, no. 1, pp. 2-14, 2020.

[5] J. Zheng and M. Fu, "A reset state estimator using an accelerometer for enhanced motion control with sensor quantization," IEEE Transactions on Control Systems Technology, vol. 18, no. 1, pp. 79-90, 2010.

[6] A. Sharon, N. Hogan, and D. E. Hardt, "The macro/micro manipulator: An improved architecture for robot control," Robotics and Computer-Integrated Manufacturing, vol. 10, no. 3, pp. $209-222,1993 . \quad$ [Online]. Available: http://www.sciencedirect.com/science/article/pii/073658459390056P

[7] J. Zheng, A. Salton, and M. Fu, "Design and control of a rotary dual-stage actuator positioning system," Mechatronics,
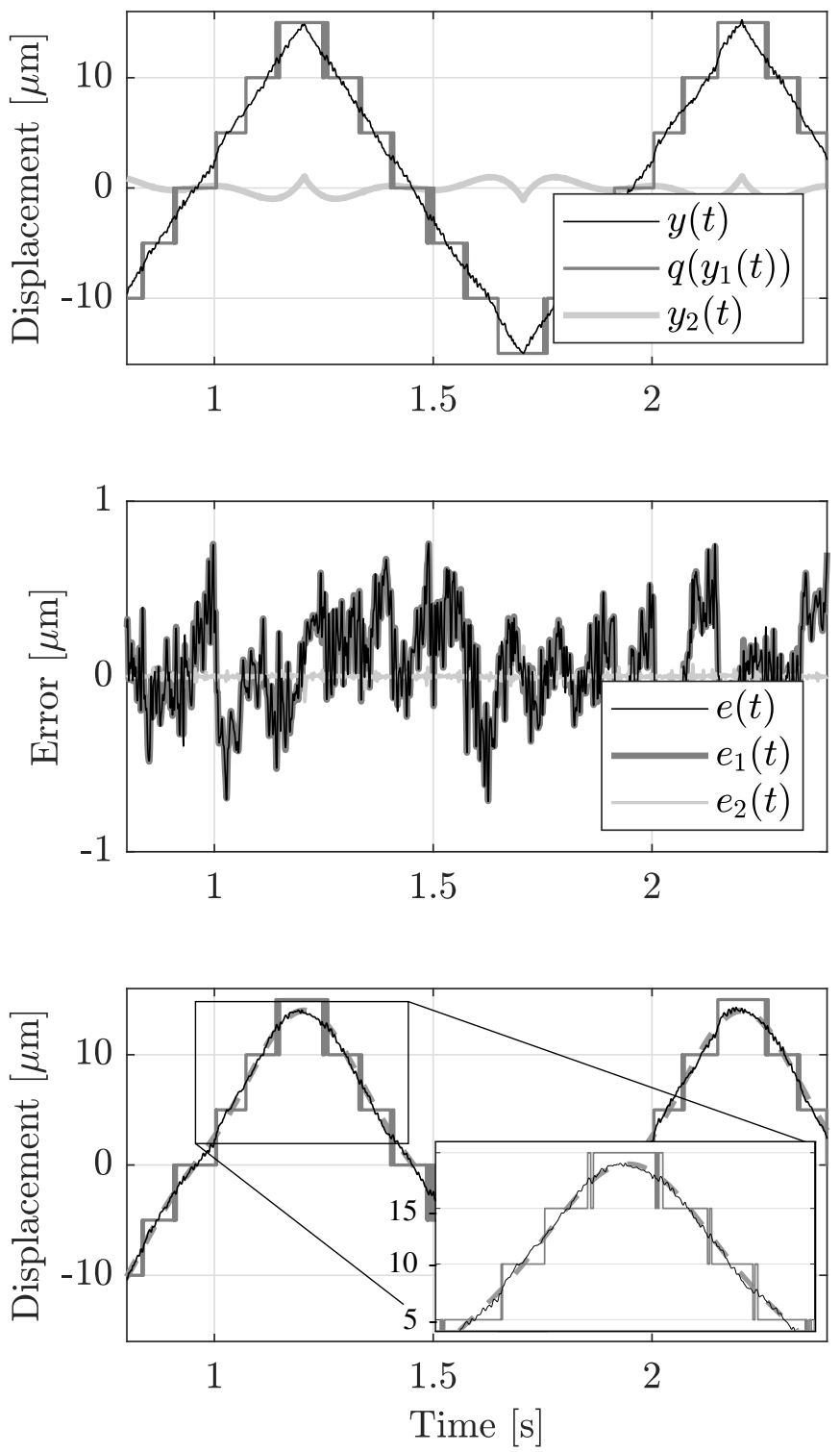

Fig. 9. Experimental results: tracking of a triangular wave. Top and middle plot are as before. Bottom plot: reference $r_{1}(t)$, the quantized output $q\left(y_{1}(t)\right.$ used for feedback, and the actual position of $y_{1}(t)$. The overall tracking error is well within the $\pm 1 \mu \mathrm{m}$ range, despite the sensor quantization of $\delta=5 \mu \mathrm{m}$.

vol. 21, no. 6, pp. 1003 - 1012, 2011. [Online]. Available: http://www.sciencedirect.com/science/article/pii/S0957415811000560

[8] J. Zheng and M. Fu, "Nonlinear feedback control of a dual-stage actuator system for reduced settling time," IEEE Transactions on Control Systems Technology, vol. 16, no. 4, pp. 717-725, 2008.

[9] K. Mori, T. Munemoto, H. Otsuki, Y. Yamaguchi, and K. Akagi, "A dual-stage magnetic disk drive actuator using a piezoelectric device for a high track density," IEEE Transactions on Magnetics, vol. 27, no. 6, pp. 5298-5300, 1991.

[10] C. Schindlbeck, C. Pape, and E. Reithmeier, "Process-integrated state estimation of optical systems with macromicro manipulators based on wavefront filtering," IEEE Robotics and Automation Letters, vol. 4, no. 4, pp. 4078-4085, 2019.

[11] T. Chen, J. Lou, Y. Yang, Z. Ren, and C. Xu, "Vibration suppression of a high-speed macro-micro integrated system using computational optimal control," IEEE Transactions on Industrial Electronics, pp. 1-1, 2019.

[12] X. Chen, X. Chen, W. Bai, and Z. Guo, "Event-triggered optimal 
control for macro-micro composite stage system via single-network adp method," IEEE Transactions on Industrial Electronics, pp. 1-1, 2020.

[13] J. van Zundert, T. Oomen, J. Verhaegh, W. Aangenent, D. J. Antunes, and W. P. M. H. Heemels, "Beyond performance/cost tradeoffs in motion control: A multirate feedforward design with application to a dualstage wafer system," IEEE Transactions on Control Systems Technology, vol. 28 , no. 2, pp. 448-461, 2020.

[14] A. T. Salton, M. Fu, J. V. Flores, and J. Zheng, "High precision over long range: a macro-micro approach to quantized positioning systems," IEEE Transactions on Control Systems Technology (submitted), 2020.

[15] K. Cai, Z. Deng, C. Peng, and K. Li, "Suppression of harmonic vibration in magnetically suspended centrifugal compressor using zero-phase odd-harmonic repetitive controller," IEEE Transactions on Industrial Electronics, vol. 67, no. 9, pp. 7789-7797, 2020.

[16] Y. Jian, D. Huang, J. Liu, and D. Min, "High-precision tracking of piezoelectric actuator using iterative learning control and direct inverse compensation of hysteresis," IEEE Transactions on Industrial Electronics, vol. 66, no. 1, pp. 368-377, 2019.

[17] Keliang Zhou, Kay-Soon Low, D. Wang, Fang-Lin Luo, Bin Zhang, and Yigang Wang, "Zero-phase odd-harmonic repetitive controller for a single-phase pwm inverter," IEEE Transactions on Power Electronics, vol. 21, no. 1, pp. 193-201, 2006. 